\title{
Does temperature constrain diet choice in a marine herbivorous fish?
}

\author{
Jethro S. Johnson ${ }^{1,4}\left([) \cdot\right.$ David Raubenheimer $^{2}$ (1) $\cdot$ Sarah J. Bury ${ }^{3} \cdot$ Kendall D. Clements ${ }^{1}([)$
}

Received: 10 April 2019 / Accepted: 11 March 2020

○) Springer-Verlag GmbH Germany, part of Springer Nature 2020

\section{Abstract}

The temperature constraint hypothesis (TCH) states that low water temperatures constrain the efficiency of algal digestion $\mathbf{A Q 1}$ in fishes to an extent that limits the viability of herbivory. It is a widely cited explanation for the decline in diversity and abundance of marine herbivorous fishes at high latitudes. A key prediction of this hypothesis is that temperature-related physiological constraints may be ameliorated in free-living herbivores by a shift from herbivory to omnivory. We investigated this prediction by examining the diet of the primarily herbivorous marine fish Odax pullus (Labridae) at multiple locations spanning a gradient of $10^{\circ}$ latitude and encompassing a change in median annual sea surface temperature of 5-7 ${ }^{\circ} \mathrm{C}$. Foregut content and stable isotope analyses were used to compare diet across locations and results were evaluated against the prediction that a latitudinal temperature constraint on herbivory would result in significant variation in the composition of adult diets or the timing of an ontogenetic switch from omnivory to herbivory at colder, higher latitudes. We found that neither the proportion of animal material consumed nor ontogenetic changes in diet varied across the distributional range of this species in a manner consistent with the predictions of the TCH. This study demonstrates that water temperature does not constrain herbivory in a marine ectothermic herbivore and thus challenges a widely cited hypothesis.

\section{Introduction}

The global distribution of marine herbivorous fishes is characterized by a decline in species richness and abundance with increasing latitude (Choat 1991; Ebeling and Hixon 1991; Harmelin-Vivien 2002; Ferreira et al. 2004; Floeter

\section{Responsible Editor: C. Trueman.}

Reviewed by undisclosed experts

Electronic supplementary material The online version of this article (https://doi.org/10.1007/s00227-020-3677-z) contains supplementary material, which is available to authorized users.

Jethro S. Johnson

jethro.johnson@jax.org

1 School of Biological Sciences, University of Auckland, Private Bag 92019, Auckland, New Zealand

2 Charles Perkins Centre, School of Life and Environmental Sciences, University of Sydney, Johns Hopkins Drive, Camperdown, Sydney, Australia

3 National Institute of Water and Atmospheric Research Ltd (NIWA), 301 Evans Bay Parade, Hataitai, PO Box 14-901, Wellington, New Zealand

4 The Jackson Laboratory for Genomic Medicine, 10 Discovery Drive, Farmington, CT 06032, USA et al. 2004, 2005). While several hypotheses have been proposed to explain this trend, one of the more widely accepted is the temperature constraint hypothesis (TCH), which states that low temperatures associated with increasing latitude constrain the efficiency of digestion by marine ectothermic herbivores (Gaines and Lubchenco 1982; Floeter et al. 2005; Behrens and Lafferty 2007).

The TCH is fundamentally a hypothesis based on physiological mechanism (Gaines and Lubchenco 1982). As such, it has been used as a proximate (sensu Mayr 1961; MacDougall-Shackleton 2011) explanation for changes in feeding behaviour, in which the ingestion of a smaller proportion of plant material is associated with declining temperature (Behrens and Lafferty 2007, 2012; Goldenberg and Erzini 2014; Vejrikova et al. 2016). In field studies, such behaviour has been used to explain decline in herbivore grazing intensity, observed with increasing latitude (Longo et al. 2014, 2018; Verges et al. 2014; Franco et al. 2015).

Global studies of biodiversity have cited the TCH as a proximate cause of latitudinal or sea surface temperature (SST)-related decline in the taxonomic diversity of herbivorous reef fishes (Harmelin-Vivien 2002; Floeter et al. 2005; Gonzalez-Bergonzoni et al. 2012) as well as changes in the functional diversity of different herbivore feeding strategies (Bender et al. 2013; Longo et al. 2014, 2018). Similarly, the 
TCH has been cited as a possible factor in shaping latitudinal and SST-related changes in algal assemblages (Poore et al. 2012; Keith et al. 2014). Finally, it has also been cited as a reason for the observation that changes in global climate may lead to range expansion by tropical herbivorous fish species and consequential destruction of macroalgal habitats (Mora 2008; Verges et al. 2014, 2016).

While the TCH is increasingly used to explain ecological and evolutionary trends, there remain very few studies that address this hypothesis at the mechanistic level at which it was originally proposed (Behrens and Lafferty 2007, 2012; Trip et al. 2014), for example, by directly examining the effect of temperature on diet and digestion in marine herbivorous fishes. We therefore undertook a detailed analysis of the diet of the New Zealand Butterfish Odax pullus, an odacine labrid that is endemic to the coastal waters of New Zealand.

Odax pullus has a natural range that encompasses $\sim 15^{\circ}$ of latitude $\left(34.4^{\circ} \mathrm{S}-49.5^{\circ} \mathrm{S}\right.$ ) (Gomon et al. 2015), and year-round sea surface temperatures (SST) below the $20{ }^{\circ} \mathrm{C}$ threshold that has been previously been hypothesized to constrain herbivory in fishes (Chiswell 1994; Floeter et al. 2005). A recent study of the demography of this species found no evidence that nutrient stress constrains patterns of growth and maturation at high latitudes (Trip et al. 2014). Therefore, according to the $\mathrm{TCH}$, this species must alter its diet to mitigate a temperature constraint on digestion of algae. Alternatively, $O$. pullus contradicts this hypothesis and is a successful herbivore at latitudes above those predicted to limit ectothermic herbivory (Floeter et al. 2005).

In the northern extent of this range $\left(\sim 36^{\circ} \mathrm{S}\right), O$. pullus, like many herbivorous fishes, is omnivorous during early development and exclusively herbivorous as an adult, consuming predominantly fucalian and laminarian algae (Clements and Choat 1993; Johnson et al. 2017). In addition, its jaw and gut morphology (Clements and Bellwood 1988) and a diverse intestinal microflora (Clements 1991; Clements et al. 2007) indicate that it is highly adapted for the digestion of plant material via microbially mediated hindgut fermentation (Mountfort et al. 2002).

We used examination of foregut contents together with stable isotope analysis to compare the diet of $O$. pullus at three locations spanning a broad latitudinal gradient. While diet analysis provides a detailed insight into very recent feeding behaviour (Hyslop 1980), stable isotope analysis, in particular $\delta^{15} \mathrm{~N}$ values, can provide a more temporally integrated perspective on the trophic level at which an animal is foraging (Dalerum and Angerbjörn 2005).

In combining these complementary approaches, our aim was to determine whether the diet of $O$. pullus changes with increasing latitude in a manner consistent with a temperature constraint on the digestion of algal material. The approach taken was twofold. First, adult diet was examined to determine whether fish at high latitudes demonstrated a change in diet-for example, increased consumption of animal material - that might reflect a change in digestive mechanism at low temperatures. Second, the size of the ontogenetic diet shift from omnivory to herbivory was compared across locations to determine whether fish at high latitude switch to an herbivorous diet at a larger size and, consequently, a more favourable gut mass: body size ratio.

\section{Materials and methods}

\section{Study sites}

Fish were collected from three locations within New Zealand: the Hauraki Gulf $\left(36.13^{\circ} \mathrm{S}-37.14^{\circ} \mathrm{S}, 174.40^{\circ}\right.$ E-175.32 $\left.{ }^{\circ} \mathrm{E}\right)$; D'Urville Island $\left(40.70^{\circ} \mathrm{S}-40.75^{\circ} \mathrm{S}, 173.97^{\circ}\right.$ $\left.\mathrm{E}-173.99^{\circ} \mathrm{E}\right)$; and Stewart Island $\left(46.54^{\circ} \mathrm{S}-47.91^{\circ} \mathrm{S}\right.$, $\left.168.10^{\circ} \mathrm{E}-168.24^{\circ} \mathrm{E}\right)$. Collectively, these locations spanned $12^{\circ}$ of latitude and a year-round mean temperature difference of approximately $5.5^{\circ} \mathrm{C}$ (Supplementary Fig. 1). A breakdown of seasonal temperatures during the study period is provided in Supplementary Table 1.

At all three locations, sampling took place at multiple shallow, temperate rocky reef sites dominated by brown algae of the orders Laminariales and Fucales. Principal Fucalian algae were Carpophyllum spp. and Landsburgia quercifolia. At the two northern locations, principal Laminarian algae were Ecklonia radiata and Lessonia variegata, while the southern location (Stewart Island) also consisted of dense stands of the giant kelp Macrocystis pyrifera. For a more detailed description of algal assemblages and details of the benthic community structure at each location see Shears and Babcock (2007).

\section{Sample collection}

Specimens of $O$. pullus used for diet analysis were collected during the austral summer (December-February). Sampling within the Hauraki Gulf occurred between January 2006 and February 2008, at D'Urville Island during January/February 2007 and February 2008, and at Stewart Island during December 2006 and December 2007.

Specimens of $O$. pullus used for stable isotope analysis were collected as above at the two southern locations (D'Urville Island and Stewart Island) and year round from the Hauraki Gulf from January 2006 to February 2008. Preliminary investigation found no evidence of seasonal variation in the nitrogen isotope values of muscle tissue collected (Supplementary Fig. 2). As a result, all stable isotope data from the Hauraki Gulf were pooled for use in subsequent comparisons. 
Fish were collected by snorkelling, using either a hand spear or speargun, and removed to a nearby boat for processing. Weight and length measurements were then recorded before the gastro-intestinal (GI) tract was removed, cleaned and divided into five equal-sized sections following (Mountfort et al. 2002). The contents of the first gut section (henceforth, referred to as "foregut") were then placed in an individually labelled polypropylene tube and frozen in liquid nitrogen. For stable isotope analysis, a small amount of dorsal white muscle tissue was removed, placed in a microcentrifuge tube and frozen in liquid nitrogen.

At all three locations, fish were sampled across as broad a size range as possible. Individuals were excluded from the study if any damage to the foregut was detected as a consequence of spearing.

Representative samples of two commonly consumed diet items were also collected from each location. For Stewart Island, Macrocystis pyrifera thallus tissue $(n=5)$ and Carpophyllum maschalocarpum reproductive tissue $(n=5)$ were collected during fieldwork. For the Hauraki Gulf, Ecklonia radiata thallus $(n=10)$ and $C$. maschalocarpum reproductive tissue $(n=10)$ were collected during a prior study (Johnson et al. 2012). For D'Urville Island, Ecklonia radiata thallus $(n=5)$ and Carpophyllum spp. reproductive tissue $(n=5)$ were taken from the anterior foregut of fish collected as part of this study. Previous work has shown that the $\delta^{15} \mathrm{~N}$ and $\delta^{13} \mathrm{C}$ values of these tissues does not alter following ingestion (Johnson et al. 2012).

\section{Sea surface temperature}

Sea surface temperature (SST) data were downloaded from the NOAA OI SST V2 High Resolution Dataset (Reynolds et al. 2007). Daily temperature measurements were extracted for the months during which sampling occurred at one or more location (January-February 2006, December 2006, January-February 2007, December 2007, January-February 2008). For comparison, daily temperature measurements were also extracted for the austral winter (June-August) for 2006, 2007, and 2008. For each study location, three commonly visited sites were selected (Supplementary Table 1) and the available SST measurement closest to each site was pooled across the dates mentioned to generate a site-specific estimate.

\section{Diet analysis}

Diet analysis was carried out as described previously (Johnson et al. 2017). Briefly, representative samples of foregut contents were thawed and examined under a stereoscopic microscope (10-50 $\times$ magnification). Material was divided into morphotype categories based on distinguishable characteristics and the proportional contribution (by mass) of each category to the diet was calculated. Each morphotype was subsequently identified to the lowest possible taxonomic resolution using algal specimens and with reference to the relevant literature (Morton and Miller 1973; Adams 1994).

\section{Stable isotope analysis}

Samples of white muscle tissue were lyophilised and cryogenically ground using an MM301 ball mill (Retsch). Lipid extraction followed Folch et al. (1957). 10-20 mg of sample was combined with $500 \mu \mathrm{l}$ chloroform, $400 \mu \mathrm{l}$ water and $1000 \mu \mathrm{l}$ methanol. Samples were vortexed and sonicated (40 kHz at $4{ }^{\circ} \mathrm{C}$ for $5 \mathrm{~min}$ ), a further $500 \mu \mathrm{l}$ of chloroform was added and samples were again sonicated, then centrifuged (2000 rcf for $5 \mathrm{~min}$ ). The chloroform layer was discarded and remaining solvent dried down in a speedvac (Thermo Savant). $0.3-0.5 \mathrm{mg}$ of lipid-extracted material was weighed into tin microcapsules and analysed using a NA 1500 Fisons elemental analyser linked to a ThermoFisher Scientific Deltaplus mass spectrometer. Stable isotope analysis was carried out at the National Institute for Water and Atmospheric Research (NIWA) stable isotope facility in Wellington, New Zealand.

\section{Data analysis}

Odax pullus has been described as a monandric protogynous hermaphrodite, with a proportion of fish transitioning from female to male at a discrete body size (Trip et al. 2011). In addition, a previous study found evidence that size-at-sexual maturity, but not size-at-sex change varied significantly across the three locations considered here (Trip et al. 2014). On this basis, fish collected in the current study were divided into four categories, chosen to reflect size and inferred reproductive status. These categories were juvenile ( $<200 \mathrm{~mm}$ FL), pre-sex-change female (201-358 mm FL), post-sex-change female ( $>358 \mathrm{~mm}$ FL) and male. The size boundary for size-at-sex change was decided based on Trip et al. (2014) and on the size of the smallest male encountered during the current study (359 mm FL). Both diet and stable isotope data were subsequently compared across reproductive categories as well as across locations. Where appropriate, comparisons of sex were also included in analysis of variance (ANOVA) type models by specifying a prior contrast between the post-sex-change female and male reproductive categories.

For statistical analysis, diet data were assigned to taxonomic categories that approximated those used in previous studies of O. pullus (Choat and Clements 1992) (Table 1). Values for each diet category were fourth root transformed, converted to resemblance matrices of Bray-Curtis dissimilarity coefficients, and subsequently analysed using Primer v6.1.12 with the Permanova + v1.0.2 add-on. Differences in 
Table 1 Percent contribution of different categories to the diet of fish from the three locations visited in this study: figures in bold are summaries of higher taxonomic data; categories with an asterisk (*) were used for statistical analysis; categories with a dagger $(\dagger)$ were combined into a single category (epiphytic rhodophyte) for inclusion in analysis

\begin{tabular}{|c|c|c|c|c|c|c|c|}
\hline Juvenile & Hauraki Gulf & D’Urville Island & Stewart Island & Intermediate female & Hauraki Gulf & D'Urville Island & Stewart Island \\
\hline $\mathrm{FL}(\mathrm{mm})$ & $132(15)$ & $128(15)$ & $137(11)$ & & $296(11)$ & $288(9)$ & $288(9)$ \\
\hline$n$ & 7 & 8 & 9 & & 16 & 15 & 18 \\
\hline Animalia & $45.75(18.37)$ & $30.6(15.27)$ & $19.41(10.74)$ & Animalia* & $1.79(0.54)$ & $10.43(3.6)$ & $2.17(1.03)$ \\
\hline Unknown_animal & & $0.02(0.02)$ & $1.26(0.65)$ & Unknown_animal & $0.16(0.16)$ & $0.04(0.04)$ & \\
\hline Annelida & & & & Annelida & & $0.24(0.18)$ & \\
\hline Arthropoda & 43 (17.29) & $23.39(14.5)$ & $5.68(2.64)$ & Arthropoda & $0.38(0.22)$ & $0.49(0.2)$ & $0.24(0.22)$ \\
\hline Bryozoa & & $3.72(2.21)$ & $0.02(0.02)$ & Bryozoa & $0.34(0.25)$ & $7.52(3.39)$ & $0.27(0.19)$ \\
\hline Cnidaria & $0.07(0.07)$ & $2.72(2.64)$ & $0.04(0.04)$ & Cnidaria & $0.65(0.31)$ & $0.6(0.3)$ & $1.15(0.85)$ \\
\hline Mollusca & $0.81(0.71)$ & $0.74(0.42)$ & $12.41(9.88)$ & Mollusca & $0.2(0.12)$ & $0.21(0.1)$ & \\
\hline Mollusca_egg & $1.87(1.87)$ & & & Mollusca_egg & $0.02(0.02)$ & $1.34(1.07)$ & $0.11(0.08)$ \\
\hline Porifera & & & & Porifera & $0.03(0.03)$ & & $0.39(0.39)$ \\
\hline Unknown_algae* & $0.28(0.28)$ & $0.06(0.06)$ & $9.38(4.97)$ & Unknown_algae* & $7.12(2.38)$ & $6.06(1.58)$ & $4.35(2.47)$ \\
\hline Chromista & & & & Chromista & & & \\
\hline Heterokontophyta & & & & Heterokontophyta & & & \\
\hline Phaeophyceae & & & & Phaeophyceae & & & \\
\hline $\begin{array}{l}\text { Unknown_phaeo- } \\
\text { phyte* }^{*}\end{array}$ & & & & $\begin{array}{l}\text { Unknown_phaeo- } \\
\text { phyte* }\end{array}$ & $4.78(3.29)$ & & \\
\hline Fucales & 34.49 (16.09) & $16.86(7.67)$ & $2.1(1.45)$ & Fucales & $17.34(4.35)$ & $19.06(2.63)$ & $10.27(4.83)$ \\
\hline Unknown_Fucales* & & & & Unknown_Fucales* & & & $5.41(4.38)$ \\
\hline Sargassaceae & & & & Sargassaceae & & & \\
\hline Carpophyllum* & $9.24(4.74)$ & $14.7(6.7)$ & $1.87(1.37)$ & Carpophyllum* & $10.4(3.08)$ & $17.59(2.55)$ & $0.51(0.25)$ \\
\hline $\begin{array}{l}\text { Carpophyllum } \\
\text { reproductives* }\end{array}$ & $25.25(12.09)$ & $2.16(2.1)$ & & $\begin{array}{l}\text { Carpophyllum } \\
\text { reproductives* }\end{array}$ & $6.93(3.61)$ & $1.47(0.59)$ & $0.77(0.45)$ \\
\hline Landsburgia* & & & & Landsburgia* & & & $0.26(0.26)$ \\
\hline Laminariales & $0.92(0.82)$ & $21.85(11.78)$ & $11.42(5.28)$ & Laminariales & $55.67(7.43)$ & $55.5(5.87)$ & $66.03(7.68)$ \\
\hline $\begin{array}{l}\text { Unknown_Laminari- } \\
\text { ales* }\end{array}$ & & & $3.46(2.63)$ & $\begin{array}{l}\text { Unknown_Laminari- } \\
\text { ales* }\end{array}$ & $2.97(1.98)$ & $3.61(2.53)$ & $7.58(2.92)$ \\
\hline Ecklonia/Lessonia* & $0.92(0.82)$ & 1.78) & $7.96(5.27)$ & Ecklonia/Lessonia* & $52.7(6.87)$ & $51.89(6.54)$ & $34.36(9.28)$ \\
\hline Macrocystis* & & & & Macrocystis* & & & $24.09(8.5)$ \\
\hline Marginariella* & & & $0.23(0.23)$ & Marginariella* & & & $3.33(2.73)$ \\
\hline Plantae & & & & Plantae & & & \\
\hline Chlorophyta* & & $0.02(0.02)$ & $1.65(1.56)$ & Chlorophyta* & & $0.05(0.04)$ & $0.35(0.33)$ \\
\hline Rhodophyta* & $18.56(13.72)$ & $30.6(15.22)$ & $56.04(11.01)$ & Rhodophyta* & $13.31(4.15)$ & $8.9(4.64)$ & $16.83(7.02)$ \\
\hline $\begin{array}{l}\text { Calcareous rhodo- } \\
\text { phyte* }\end{array}$ & & & $0.03(0.03)$ & $\begin{array}{l}\text { Calcareous rhodo- } \\
\text { phyte* }\end{array}$ & $0.01(0.01)$ & & $0.33(0.24)$ \\
\hline $\begin{array}{l}\text { Corticated rhodo- } \\
\text { phyte* }\end{array}$ & & $0.07(0.06)$ & & $\begin{array}{l}\text { Corticated rhodo- } \\
\text { phyte* }\end{array}$ & $0.32(0.22)$ & & $0.02(0.02)$ \\
\hline $\begin{array}{l}\text { Filamentous } \\
\text { rhodophyte }\end{array}$ & $14.59(10.52)$ & $26.77(14.23)$ & $1.01(0.95)$ & $\begin{array}{l}\text { Filamentous } \\
\text { rhodophyte }^{\dagger}\end{array}$ & $7.87(2.77)$ & $2.14(0.86)$ & $8.27(5.06)$ \\
\hline $\begin{array}{l}\text { Fine filamentous } \\
\text { rhodophyte }^{\dagger}\end{array}$ & & $0.01(0.01)$ & $30.71(10.74)$ & $\begin{array}{c}\text { Fine filamentous } \\
\text { rhodophyte }^{\dagger}\end{array}$ & $2.66(1.66)$ & $5.97(4.06)$ & $6.99(4.69)$ \\
\hline $\begin{array}{l}\text { Sheet-like } \\
\text { rhodophyte }^{\dagger}\end{array}$ & $3.97(3.28)$ & $3.75(1.78)$ & $24.29(8.73)$ & $\begin{array}{l}\text { Sheet-like } \\
\text { rhodophyte }^{\dagger}\end{array}$ & $2.45(2)$ & $0.79(0.68)$ & $1.23(0.72)$ \\
\hline \multirow[t]{3}{*}{ Large female } & & & & Male & & & \\
\hline & $419(12)$ & $439(13)$ & $456(11)$ & & $416(9)$ & $455(12)$ & $450(10)$ \\
\hline & 12 & 18 & 19 & & 12 & 11 & 10 \\
\hline Animalia* & $8.81(3.08)$ & $12.62(3.34)$ & $5.41(1.77)$ & Animalia* & $2.61(1.33)$ & $7.33(2.25)$ & $7.61(2.62)$ \\
\hline Unknown_animal & $1.36(0.73)$ & & & Unknown_animal & $1.23(1.23)$ & $2.28(2.2)$ & $0.1(0.1)$ \\
\hline Annelida & & $0.21(0.08)$ & & Annelida & & $0.22(0.13)$ & \\
\hline
\end{tabular}

\begin{tabular}{|l|l|l|l|l|}
\hline Journal : Large 227 & Article No : 3677 & Pages : 12 & MS Code : 3677 & Dispatch : 19-3-2020 \\
\hline
\end{tabular}


Table 1 (continued)

\begin{tabular}{|c|c|c|c|c|c|c|c|}
\hline \multicolumn{4}{|l|}{ Large female } & \multicolumn{4}{|l|}{ Male } \\
\hline Arthropoda & $0.25(0.2)$ & $0.08(0.04)$ & $0.02(0.02)$ & Arthropoda & $0.09(0.05)$ & $0.02(0.01)$ & $0.23(0.23)$ \\
\hline Bryozoa & $3.75(3.07)$ & $9.08(2.17)$ & $0.49(0.44)$ & Bryozoa & $0.52(0.52)$ & $4.74(1.52)$ & $0.39(0.36)$ \\
\hline Cnidaria & $3.37(1.61)$ & $2.33(2.19)$ & $4.14(1.75)$ & Cnidaria & $0.56(0.26)$ & $0.06(0.06)$ & $6.3(2.55)$ \\
\hline Mollusca & $0.07(0.07)$ & $0.02(0.02)$ & & Mollusca & & & $0.06(0.06)$ \\
\hline Mollusca_egg & & $0.91(0.43)$ & & Mollusca_egg & $0.21(0.21)$ & & $0.53(0.53)$ \\
\hline Porifera & & & $0.76(0.61)$ & Porifera & & & \\
\hline Unknown_algae* & $2.76(1.2)$ & $2.46(0.88)$ & $4.46(1.74)$ & Unknown_algae* & $2.59(1.32)$ & $1.53(0.82)$ & $1.89(1.26)$ \\
\hline Chromista & & & & Chromista & & & \\
\hline Heterokontophyta & & & & Heterokontophyta & & & \\
\hline Phaeophyceae & & & & Phaeophyceae & & & \\
\hline $\begin{array}{l}\text { Unknown_phaeo- } \\
\text { phyte* }^{*}\end{array}$ & $1.19(1.19)$ & & & $\begin{array}{l}\text { Unknown_phaeo- } \\
\text { phyte* }\end{array}$ & $4.39(2.16)$ & & \\
\hline Fucales & 11.87 (4.33) & $22.04(4.3)$ & $17.5(6.13)$ & Fucales & $9.58(4.08)$ & $21.46(7.98)$ & $6.71(3.9)$ \\
\hline Unkown_Fucales* & & & $3.86(3.53)$ & Unkown_Fucales* & & $0.07(0.07)$ & $3.09(2.62)$ \\
\hline Sargassaceae & & & & Sargassaceae & & & \\
\hline Carpophyllum* & $6.7(2.36)$ & $19.14(3.69)$ & $2.79(1.71)$ & Carpophyllum* & $(2.24)$ & $14.1(4.13)$ & $3.3(3.08)$ \\
\hline $\begin{array}{l}\text { Carpophyllum } \\
\text { reproductives* }\end{array}$ & $5.17(3.57)$ & $2.9(1.29)$ & $0.51(0.28)$ & $\begin{array}{l}\text { Carpophyllum } \\
\text { reproductives* }\end{array}$ & $4.17(2.79)$ & $7.28(4.75)$ & $0.32(0.21)$ \\
\hline Landsburgia* & & & & Landsburgia* & & & \\
\hline Laminariales & 62.24 (7.79) & $61.04(5.95)$ & 59.51 (7.93) & Laminarial & $78.17(5.49)$ & $68.33(8.26)$ & $\begin{array}{r}82.47 \\
(5.77)\end{array}$ \\
\hline $\begin{array}{l}\text { Unknown_Laminari- } \\
\text { ales* }\end{array}$ & & $1.15(0.56)$ & $1.63(0.82)$ & aminari- & $2.21(1.55)$ & $0.92(0.44)$ & $7.13(2.95)$ \\
\hline Ecklonia/Lessonia* & $62.24(7.79)$ & $59.89(5.83)$ & $20.49(7.31)$ & Ecklonia/Lessonia* & $75.97(5.32)$ & $67.42(8.16)$ & $\begin{array}{r}13.67 \\
(7.25)\end{array}$ \\
\hline Macrocystis* & & & $37.4(7.78)$ & Macrocystis* & & & $\begin{array}{r}61.67 \\
\quad(6.94)\end{array}$ \\
\hline Marginariella* & & & $10.35(5.63)$ & Marginariella* & & & $0.01(0.01)$ \\
\hline Plantae & & & & Plantae & & & \\
\hline Chlorophyta* & & $0.59(0.45)$ & $0.3(0.27)$ & Chlorophyta* & & $0.07(0.05)$ & \\
\hline Rhodophyta* & $13.12(6.13)$ & $1.25(0.62)$ & $12.82(3.69)$ & Rhodophyta* & $2.65(1.28)$ & $1.28(0.87)$ & $1.31(0.91)$ \\
\hline $\begin{array}{l}\text { Calcareous rhodo- } \\
\text { phyte* }\end{array}$ & $3.53(3.53)$ & & $1.52(1.02)$ & $\begin{array}{l}\text { Calcareous rhodo- } \\
\text { phyte* }\end{array}$ & $0.05(0.04)$ & & $0.24(0.17)$ \\
\hline $\begin{array}{l}\text { Corticated rhodo- } \\
\text { phyte* }\end{array}$ & $0.79(0.76)$ & $0.08(0.07)$ & $0.03(0.03)$ & $\begin{array}{l}\text { Corticated rhodo- } \\
\text { phyte* }\end{array}$ & $1.52(1)$ & & \\
\hline $\begin{array}{l}\text { Filamentous } \\
\text { rhodophyte }^{\dagger}\end{array}$ & $3.84(2.49)$ & $0.21(0.1)$ & $3.06(1.83)$ & $\begin{array}{l}\text { Filamentous } \\
\text { rhodophyte }^{\dagger}\end{array}$ & $0.57(0.31)$ & $0.89(0.68)$ & $0.1(0.06)$ \\
\hline $\begin{array}{l}\text { Fine filamentous } \\
\text { rhodophyte }^{\dagger}\end{array}$ & & $0.53(0.39)$ & $5.91(2.45)$ & $\begin{array}{c}\text { Fine filamentous } \\
\text { rhodophyte }^{\dagger}\end{array}$ & $0.12(0.1)$ & $0.21(0.21)$ & $0.94(0.94)$ \\
\hline $\begin{array}{l}\text { Sheet-like } \\
\text { rhodophyte }\end{array}$ & $(2.58)$ & $0.43(0.27)$ & $2.3(1.7)$ & $\begin{array}{l}\text { Sheet-like } \\
\text { rhodophyte }^{\dagger}\end{array}$ & $0.39(0.21)$ & $0.18(0.12)$ & $0.04(0.03)$ \\
\hline
\end{tabular}

Numbers in parentheses give standard error

diet composition across reproductive categories and across locations were tested using permutational multivariate analysis of variance (PERMANOVA) and assumptions regarding homogeneity of dispersion were tested using permutational dispersion tests (PERMDISP).

Statistical analysis of nitrogen stable isotope values $\left(\delta^{15} \mathrm{~N}\right)$ was performed using univariate ANOVA in R v2.15.2 (Team 2012). Depending on whether sex was included in models, size was either treated as a continuous variable or treated as a discrete factor in the form of the reproductive categories described above.

\begin{tabular}{|l|l|l|l|l|}
\hline Journal : Large 227 & Article No : 3677 & Pages : 12 & MS Code : 3677 & Dispatch : 19-3-2020 \\
\hline
\end{tabular}




\section{Results}

\section{Sea surface temperature}

Median SST, plus inter-quartile ranges, at the time of sampling were $20.3{ }^{\circ} \mathrm{C}(18.7,21.0), 17.0{ }^{\circ} \mathrm{C}(16.1,17.9)$, and $13.6^{\circ} \mathrm{C}(13.0,14.1)$ for the Hauraki Gulf, D'Urville Island, and Stewart Island, respectively. By contrast, median winter (July-September) SSTs for the years sampled was $15.3{ }^{\circ} \mathrm{C}(14.5,16.2), 12.9^{\circ} \mathrm{C}(12.3,13.9)$, and $10.7{ }^{\circ} \mathrm{C}(10.4,11.4$, Supplementary Fig. 1, Supplementary Table 1).

\section{Diet analysis}

Foregut contents were examined for 155 specimens of O. pullus collected from the three locations in this study (Hauraki Gulf: $n=47$, D'Urville Island: $n=52$, Stewart Island: $n=56)$ and PERMANOVA tests revealed significant changes in diet across locations $(p($ perm $)=0.0001)$, and with ontogeny $(p$ perm $)=0.0001)$, as well as an interaction between these terms $(p($ perm $)=0.0001$, Supplementary Table 2A).

Diet data for adult fish indicated they were herbivorous regardless of their location or reproductive classification (Table 1) and principal co-ordinate analysis (PCO) indicated that differences in diet were driven by relative consumption of different species of laminarian algae (Fig. 1). In particular, adult fish at Stewart Island ingested a large proportion of Macrocystis pyrifera; this alga was absent from the diets of fish at the other two locations, which instead consumed members of the family Lessoniaceae.

Analysis of the dispersion of Bray-Curtis distance measures was significantly different across locations (Supplementary Table 2A), with PCO indicating that the trend in dispersion was driven by greater variation in diet at Stewart Island (Fig. 1). As significance in PERMANOVA tests is susceptible to differences in dispersion (Anderson and Walsh 2013), it is likely that the significant variation in diet composition reported here reflects differences in the extent to which diets vary within locations as well as variation between locations.

At all three locations, juveniles consumed a greater proportion of animal material and rhodophyte algae than adult fish (Table 1). Pairwise PERMANOVA supported this observation and, in addition, showed no significant difference in the composition of juvenile diets across locations (Supplementary Table 2, Supplementary Fig. 3).

The size range of juvenile fish sampled during the austral summer was limited (Table 1a) and foregut content data were therefore insufficient to assess whether fish at high latitude make the switch to herbivory at a larger body size. Nonetheless, by the time they had reached sexual maturity, fish collected at all three locations were exclusively herbivorous (Table 1).
Fig. 1 Principal co-ordinate analysis plot of Bray-Curtis dissimilarity between adult diets, following fourth root transformation of the categories depicted in Table 1. Data are for intermediate females (small circles), large females (large circles), and males (triangles), and for the Hauraki Gulf (blue), D'Urville Island (red), and Stewart Island (green). Overlaid vectors depict strength of the Pearson correlation between particular diet categories and the position of samples along the PCO axes shown

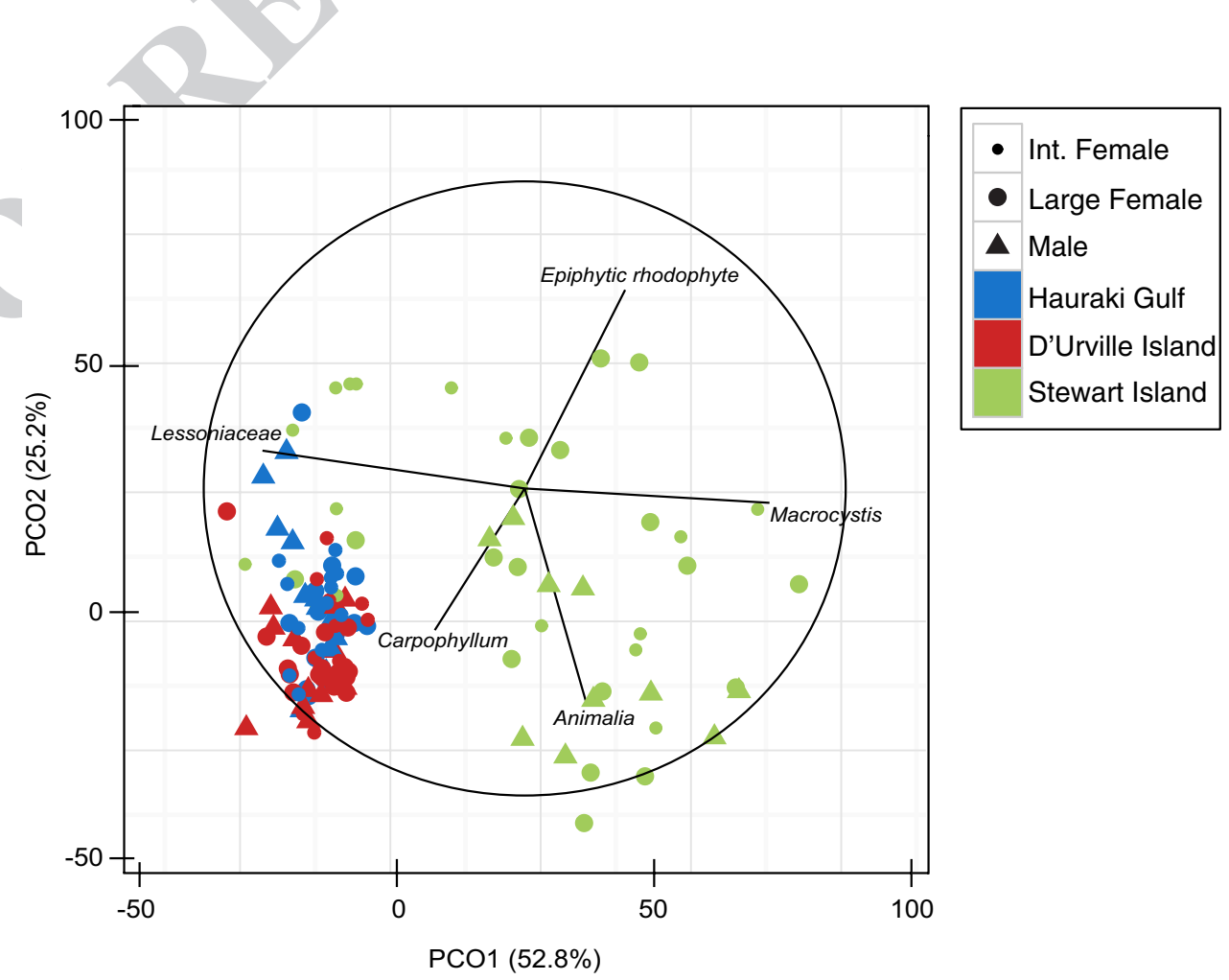




\section{Stable isotope analysis}

Stable isotope $\left(\delta^{15} \mathrm{~N}\right.$ and $\left.\delta^{13} \mathrm{C}\right)$ values were measured for 188 fish and for the two most common diet items consumed at each location. Those diet items that were comparable (Carpophyllum spp. and Ecklonia radiata) showed minimal variation in $\delta^{15} \mathrm{~N}$ values across locations $(<1 \%$ ), but greater variation in $\delta^{13} \mathrm{C}$ values, particularly at Stewart Island (Fig. 2). Due to the fact that $\delta^{13} \mathrm{C}$ values had less utility as an indicator of trophic level, subsequent analysis primarily focussed on $\delta^{15} \mathrm{~N}$.

Mean $\delta^{15} \mathrm{~N}$ discrimination values $\left(\Delta^{15} \mathrm{~N}\right)$ between plant and adult fish tissues were $3.66( \pm 0.68 \mathrm{SE}), 1.89( \pm 0.98$ $\mathrm{SE})$, and $2.72( \pm 1.04 \mathrm{SE})$, for the Hauraki Gulf, D'Urville Island, and Stewart Island, respectively. At all three locations, juvenile $\delta^{15} \mathrm{~N}$ values were higher than those observed in adult fish, which, in concordance with diet data, indicated a greater contribution of animal tissue to juvenile diets. The $\Delta^{15} \mathrm{~N}$ between juvenile and pre-sex-change females was high at D'Urville Island $(1.74 \pm 0.26 \mathrm{SE})$ and the Hauraki Gulf $(1.28 \pm 0.15 \mathrm{SE})$, when compared to Stewart Island $(0.82 \pm 0.30$ SE, Fig. 2$)$.

The temporally integrated fish muscle $\delta^{15} \mathrm{~N}$ values, together with the consistency of trends observed across locations (Fig. 2), meant that it was possible to further explore the relationship between muscle tissue $\delta^{15} \mathrm{~N}$ values and ontogeny using a linear modelling approach. Preliminary models including only large (>359 mm FL), post-sex-change fish indicated a significant effect of sex on $\delta^{15} \mathrm{~N}$ values $(F=4.76, d f=1, p<0.05$, Fig. 2, Supplementary Table 3 ). Due to the inherent nesting of sex within a restricted size range, male data were excluded from subsequent models of the relationship between $\delta^{15} \mathrm{~N}$ values and size.
General linear models of $\delta^{15} \mathrm{~N}$ values based only on female data were significant for location as well as firstand second-order size coefficients ( $p<0.05$, Supplementary Table 4). To account for possible location-specific effects, such as variation in background isotope levels or variation in the magnitude of diet-tissue discrimination, the $\delta^{15} \mathrm{~N}$ value of each individual fish was adjusted by subtracting the $\delta^{15} \mathrm{~N}$ value calculated from the vertex of a population-specific second-order polynomial (Supplementary Fig. 4). The resulting data were used to compare changes in $\delta^{15} \mathrm{~N}$ value with size across populations (Fig. 3).

Comparison of adjusted data indicated that, during early development, $\delta^{15} \mathrm{~N}$ values declined less dramatically with size at Stewart Island than at the other two locations. This observation was supported by pairwise comparison between locations, which resulted in a significant $\operatorname{size}^{2} \times$ region interaction when comparing D'Urville Island and Stewart Island $(F=6.89, d f=1, p=0.011$, Supplementary Table $5 \mathrm{C}) . \delta^{15} \mathrm{~N}$ values also reached a population minimum at a smaller size at Stewart Island than at either of the other two locations (Fig. 3, Table 2).

In addition to trends reflecting an early ontogenetic switch from omnivory to herbivory, $\delta^{15} \mathrm{~N}$ values also increased with size in large female $O$. pullus, most notably at Stewart Island (Supplementary Fig. 4).

\section{Discussion}

If the hypothesis that temperature constraints on digestion are responsible for a decline in the richness and abundance of marine herbivorous fishes with increasing latitude (Floeter et al. 2005) is correct, then this requires one of two possible scenarios: (i) that fish at high latitudes alter their
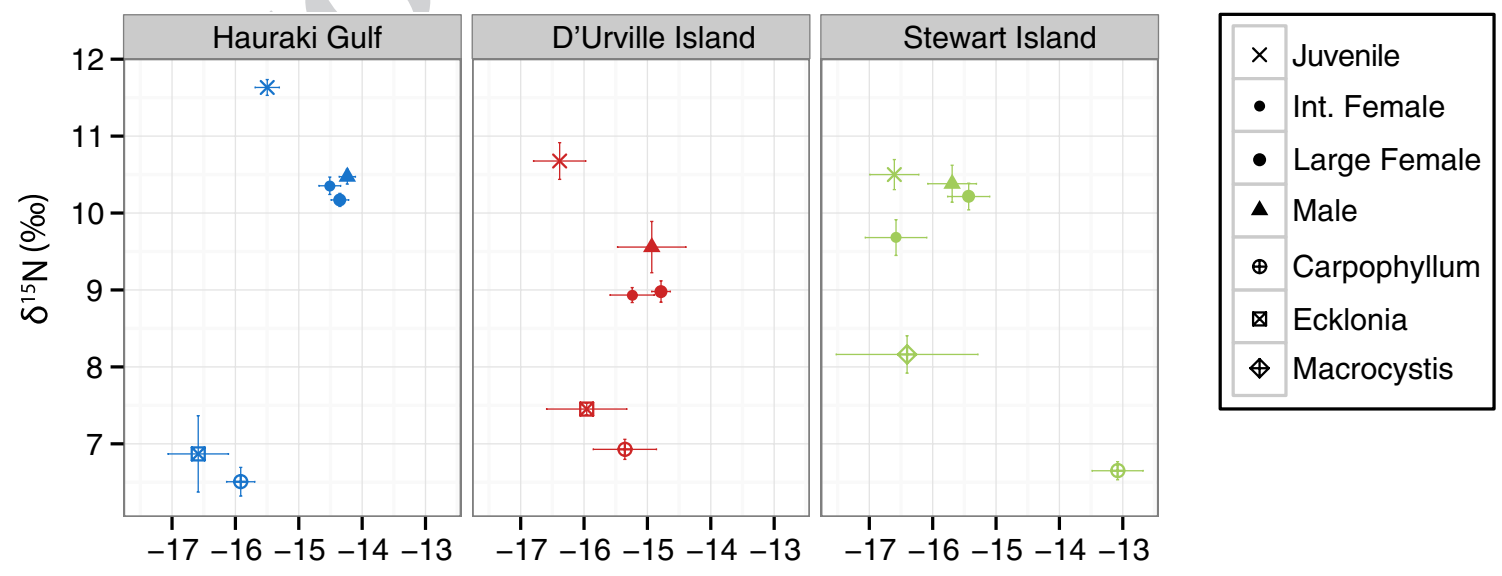

$\delta^{13} \mathrm{C}(\% 0)$

Fig. 2 Relationship between the $\delta^{15} \mathrm{~N}$ and $\delta^{13} \mathrm{C}$ values of diet items and fish white muscle tissue collected from the three locations considered in this study. Error bars depict standard error 
Fig. 3 Second-order polynomial regression of the relationship between size (fork length) and deviation of $\delta^{15} \mathrm{~N}$ value from the population minimum estimated for each location (see "Methods"). Regressions are: Hauraki Gulf (blue), D’Urville Island (red), and Stewart Island (green). Data points are: Hauraki Gulf (circle), D'Urville Island (square), and Stewart Island (triangle). Data are for female fish only. Shaded areas indicate $95 \%$ confidence intervals. Estimated sizes (FL) at the minimum $\delta^{15} \mathrm{~N}$ for each population were as follows Hauraki Gulf: 398 mm, D’Urville Island: $395 \mathrm{~mm}$, Stewart Island: $340 \mathrm{~mm}$ (Table 2)

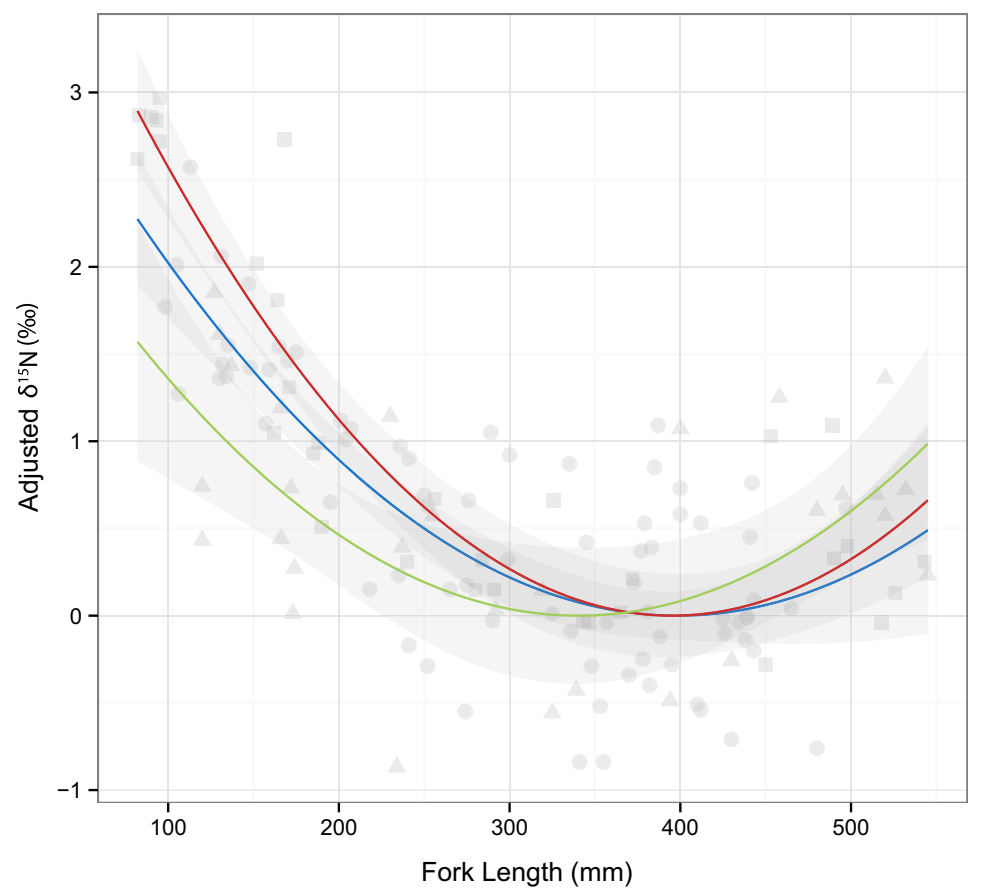

Table 2 Estimated size and age at population minima for white muscle tissue $\Delta^{15} \mathrm{~N}$ value: length estimates (FL) are derived from the population vertices depicted in Fig. 3; age estimates are derived from the size-age relationships estimated by Trip et al. (2011); mass estimates (gutted weight) are derived from a linear regression of size vs. $\sqrt[3]{\text { mass }}$

\begin{tabular}{|c|c|c|c|c|c|}
\hline \multirow[t]{2}{*}{ Location } & \multirow[t]{2}{*}{$\begin{array}{l}\text { Fork } \\
\text { length } \\
(\mathrm{mm})\end{array}$} & \multicolumn{2}{|c|}{$\begin{array}{l}\text { Fork } \\
\text { length } \pm \text { std } \\
\text { error }\end{array}$} & \multirow[t]{2}{*}{$\begin{array}{l}\text { Approxi- } \\
\text { mate age } \\
\text { (years) }\end{array}$} & \multirow[t]{2}{*}{$\begin{array}{l}\text { Approxi- } \\
\text { mate mass } \\
\text { (g) }\end{array}$} \\
\hline & & - & + & & \\
\hline Hauraki Gulf & 398 & 439 & 372 & 3.5 & 753 \\
\hline D'Urville Island & 395 & 426 & 37 & & 734 \\
\hline Stewart Island & 340 & 376 & 321 & 5 & 447 \\
\hline
\end{tabular}

diet in the face of declining temperature (e.g. Behrens and Lafferty 2012) or (ii) that they suffer the physiological and demographic consequences of nutrient stress (Trip et al. 2014). Following detailed analysis of the diet of $O$. pullus across a broad latitudinal gradient $\left(36^{\circ} \mathrm{S}-47^{\circ} \mathrm{S}\right)$, we found no evidence that this species reduced the extent of its herbivory at high latitudes. Likewise Trip et al. (2014) found no evidence that the demography of this species was altered in a manner consistent with the TCH. Taken together, these studies provide a unique, species-level analysis that provides no evidence to support the hypothesis of a temperature constraint on herbivory.

This study examined the diet and stable isotope ecology of a single species to address a physiological hypothesis and, in doing so, challenge assumptions about global trends in biodiversity and evolution. While the multiple levels at which the $\mathrm{TCH}$ is being evoked may appear confusing, we argue that addressing a physiological hypothesis with an ecological study is valid because different levels of analysis are beneficial and mutually informative (MacDougall-Shackleton 2011). Previous examinations of the TCH have either been conducted at the ecosystem level (e.g. Floeter et al. 2005; Longo et al. 2018); on omnivores, rather than herbivores (Behrens and Lafferty 2007, 2012); or have relied on minimally invasive observations such as taxonomic counts or bite rates (e.g. Verges et al. 2014; Longo et al. 2018), which may be of limited value when it comes to inferring the quality of diets (Clements et al. 2009). A detailed examination of the $\mathrm{TCH}$ in a free-living marine herbivorous fish has therefore been lacking.

While evidence derived from a single species does not refute a global hypothesis, it does highlight constraints that need to be considered at all levels. The absence of temperature-related changes in the diet of a species that may reasonably be expected to be subjected to the physiological constraints of the TCH highlights that much still needs to be learned about the diet and digestive physiology of marine herbivorous fishes. Closing this knowledge gap is necessary to evaluate the true potential for temperature to constrain the physiological processes underlying ectothermic herbivory.

\section{Latitudinal variation in diet inferred through examination of ingested material}

At all three locations visited during this study, juvenile $O$. pullus were omnivorous, whereas adult $O$. pullus predominantly ingested a balance of laminarian and fucalian algae,

\begin{tabular}{|l|l|l|l|l|}
\hline Journal : Large 227 & Article No : 3677 & Pages : 12 & MS Code : 3677 & Dispatch : 19-3-2020 \\
\hline
\end{tabular}


with a small but consistent contribution from rhodophyte and animal taxa. The composition of the rhodophyte and animal taxa ingested supports our field observations that most of the material contributing to these two categories were epibionts on phaeophyte macroalgae. While these items featured prominently in the diet of juvenile fish, it is not clear that they were being actively targeted by adult fish due to the fact that consumption of such epibionts may be incidental.

Epiphytic rhodophytes, as well as animal material, may be expected to have higher protein content than phaeophytes (Johnson et al. 2017). In consequence, increased consumption of either of these categories, whether intended or not, would indicate a shift to a high protein diet, which could in turn be interpreted as evidence to support reduced efficiency of herbivorous digestion (Behrens and Lafferty 2007). Similarly, large differences in the structural and storage carbohydrates of phaeophyte and rhodophyte algae (Lee 2008) mean that increased consumption of the latter could also reflect underlying changes in carbohydrate digestion. The fact that neither of these diet categories altered appreciably across locations may therefore be taken to indicate that the digestive mechanism of $O$. pullus is likely to have remained consistent across the latitudinal range encompassed by this study.

We attribute the significant differences observed in the diet of adult fish to: (i) the extent to which diets varied between locations, specifically variation in the type of laminarian algae consumed, and (ii) the extent to which diets varied within locations. Most noticeably, fish at Stewart Island consumed the giant kelp $M$. pyrifera-a species that is restricted to cool waters (Adams 1994). It is abundant at Stewart Island, but absent from the Hauraki Gulf (Shears and Babcock 2007). Whilst it is possible that the availability of M. pyrifera at Stewart Island may contribute to the success of $O$. pullus at such high latitudes, $\mathrm{PCO}$ analysis demonstrated that a proportion of fish sampled at this location consumed a diet comparable to that observed at D'Urville Island and the Hauraki Gulf. It is therefore more likely that significant differences in diet reflected differences in the diversity of algal assemblages rather than a behavioural response in the face of changes in temperature associated with latitude. This conclusion is supported by the observation that $O$. pullus consumes the bull kelp Durvillaea antarctica elsewhere within New Zealand (Taylor and Schiel 2010) as well as the invasive species Undaria pinnatifida (Baker et al. 2016), observations which together suggest that the diversity of phaeophytes consumed by this species is greater than that reported here.

\section{Latitudinal variation in diet inferred through stable isotope analysis}

Stable isotope analysis was used in conjunction with diet analysis because it can provide a complementary, longer-term view of consumer diet and trophic status (Dalerum and Angerbjörn 2005). It is also indicative of assimilated diet as opposed to ingested diet, meaning isotope values would reflect potential variation in nutrient assimilation brought about by changes in digestive mechanism.

Preliminary investigation of white muscle tissue isotope values at one of the three locations in this study (Hauraki Gulf) revealed no evidence that $\delta^{15} \mathrm{~N}$ values varied across the year in spite of known seasonal variation in diet at this location (Clements and Choat 1993; Johnson et al. 2017). Measured isotope values were therefore assumed to provide a better reflection of year-round feeding behaviour than diet analysis, which, although carried out at a range of temperatures sufficient to test the TCH, was nonetheless restricted to the austral summer (December-February).

However, the extent to which isotope values reported in this study reflect diet during the coldest months of the year (July-September) may be limited by the fact that isotope values can be biased towards times of the year where somatic growth rates are highest (Perga and Gerdeaux 2005). A further factor that complicates the inference of diet from stable isotope analysis is the fact that the discrimination factor $\left(\Delta^{15} \mathrm{~N}\right)$ expected between diet and consumer tissues may be variable and is therefore difficult to predict (Vander Zanden and Rasmussen 2001; McCutchan et al. 2003; Caut et al. 2009).

Acknowledging the caveats mentioned above, the relative magnitude of $\Delta^{15} \mathrm{~N}$ observed at each location in this study remains a useful indicator of the degree of trophic separation between fish and algal tissues. While the $\Delta^{15} \mathrm{~N}$ between commonly consumed phaeophytes and adult tissues varied between locations, the fact that it did not increase with latitude supports the conclusion that adult fish did not increase consumption of animal material in response to declining temperature.

The difference in $\delta^{15} \mathrm{~N}$ between juvenile and adult tissues also changed with latitude and was noticeably smaller at Stewart Island than elsewhere. The greater similarity between juvenile and adult diets at this location was supported by examination of ingested material, which showed that juvenile fish from Stewart Island consumed the smallest proportion of animal material (19\%) and the largest proportion of epiphytic rhodophytes (56\%) of any location considered. In addition to contradicting the pattern predicted by the $\mathrm{TCH}$, this result supports the assertion that rhodophyte material may substitute for animal material in omnivorous diets prior to the onset of herbivory (Muñoz and Ojeda 2000; Johnson et al. 2017).

\section{The effect of temperature on the ontogenetic switch from omnivory to herbivory}

Herbivores reliant on microbially mediated mechanisms of digestion are assumed to have a greater relative gut 
capacity than omnivores or carnivores, due to a need for longer digest retention times (Stevens and Hume 1998). In recognition of this, the allometric scaling of gut capacity with body size has been suggested as a reason for the occurrence of omnivory during the early stages of development in many herbivorous fish species (White 1985). It follows that if an ontogenetic diet shift occurs at a size where relative gut capacity enables herbivory to become viable, then reduced microbial productivity as a consequence of declining temperature would necessitate a greater body size before fish are able to become herbivorous. Such a scenario could be observed across a declining temperature gradient and would provide evidence for the TCH.

Likely seasonal settlement patterns, combined with high growth rate during early development (Trip et al. 2014), meant that the size range of juvenile fish available to sample during the austral summer was limited. Consequently, it was not possible to establish the size at onset of herbivory using available foregut content data. In contrast, the time taken for a change in dietary ${ }^{15} \mathrm{~N}$ to be integrated into consumer tissues meant it was possible to use stable isotopes to model ontogenetic changes in diet reflected by changing $\delta^{15} \mathrm{~N}$ values within populations.

The incorporation of isotopes into tissues following a change in diet has traditionally been modelled using a negative exponent (Wolf et al. 2009). However, the use of polynomials in the current study reflects the fact that further ontogenetic changes in $\delta^{15} \mathrm{~N}$ values were evident (particularly at Stewart Island) that could not be attributed to the expected switch from omnivory to herbivory. Importantly, this approach still allowed us to estimate the size and age at which isotope values indicative of an herbivorous diet appear in adult tissues.

Interpretation of trends in $\delta^{15} \mathrm{~N}$ values needs to account for the fact that both growth and catabolic turnover contribute to isotope incorporation into consumer tissues (Fry and Arnold 1982, Wolf et al. 2009). This complicates interpretation of our results because (i) estimates of the relative contribution of growth vs. catabolic turnover to isotope incorporation are variable (Reich et al. 2008; Carleton and Martinez del Rio 2010), and (ii) growth and catabolic turnover may decline with temperature at different rates. In consequence, the relative contribution of these two processes to ontogenetic changes in isotope signatures at the three different study locations is difficult to predict.

Assuming growth is the major driver of isotope incorporation during early development (i.e. when growth rates are high), then a temperature constraint on the size at onset of herbivory at high latitudes would be reflected by an increase in the size at which an herbivorous isotope signature is fully incorporated into fish tissue. No such trend was observed for $O$. pullus. Instead, fish in colder waters incorporated an herbivorous isotope signature at a smaller body size - a result that is inconsistent with the predictions of the TCH.

The completion of an ontogenetic diet shift at a smaller body size at Stewart Island likely reflects the fact that juvenile diets at this location contained less animal material and were isotopically more similar to adult diets than at either of the two warmer locations. However, it may also be that the shift to herbivory is influenced by age, or seasonal effects, such as the availability of specific diet items (Johnson et al. 2017). If this were the case, then fish at Stewart Island would switch diets at a smaller body size due to the slower growth rates encountered at this location (Trip et al. 2014). Finally, it may be that catabolic turnover makes a greater relative contribution to isotope incorporation at Stewart Island where growth rates are slower. This last scenario would occur if the decline in catabolic turnover with temperature was slower than the decline in growth rate observed by Trip et al. (2014).

\section{$\delta^{15} \mathrm{~N}$ values of male and large female fish}

$\delta^{15} \mathrm{~N}$ values in the tissues of male and large female $O$. pullus showed variation beyond that expected as a result of the early ontogenetic switch from omnivory to herbivory. Analysis of foregut contents showed no clear trend for increased consumption of animal material either with size or sex; however, these changes may nonetheless reflect alterations in diet or habitat. The fact that in female fish this trend was strongest at Stewart Island suggests that it may be explained by the greater habitat diversity at this location. Of the two plant tissues sampled at this location, $M$. pyrifera had a noticeably higher mean $\delta^{15} \mathrm{~N}$ value and it may therefore be that larger fish are more likely to migrate from shallow reef environments dominated by $E$. radiata to deeper water containing $M$. pyrifera stands.

\section{Conclusion}

In conclusion, following a detailed examination of the diet of O. pullus across sites that span a broad latitudinal gradient, no evidence was found to support the TCH. When combined with the fact that the demography of $O$. pullus shows no nutritional constraint at high latitude (Trip et al. 2014), this study brings into question the scope and validity of the widely cited hypothesis that latitudinal gradients of temperature limit the distribution of marine herbivorous fishes.

The success of $O$. pullus at temperatures below those cited as having the potential to limit herbivorous mechanisms of digestion has been attributed to a highly specialised diet and digestive physiology (Floeter et al. 2005). However, the presence of other marine herbivorous fish species at high latitudes that vary in diet and digestive mechanism (Iken

\section{照 Springer}


et al. 1997; Hickerson and Cunningham 2005; Clements et al. 2009; Ruz et al. 2018) contradicts this. The general lack of understanding of nutrition in herbivorous fishes and, in particular, how digestive mechanisms respond in the face of changes in temperature, means that much stands to be gained from further study of the nutritional ecology of $O$. pullus and other temperate herbivorous fish species.

Acknowledgements The authors would like to thank Elizabeth Trip for help with all stages of this work; Brady Doak, Murray Birch, Martin Holmes, Rob Swale and Schannel van Dijken for help with sample collection; and three anonymous reviewers for their comments on improving the manuscript. This study was supported by a Royal Society of New Zealand Marsden grant awarded to D.R., K.D.C., and a Commonweaith Scholarship and Fellowship Plan doctoral scholarship awarded to J.S.J.

Author contributions KDC and DR conceived and supervised study; KDC, DR and JSJ collected samples; JSJ performed diet analysis; SJB and JSJ performed stable isotope analysis. JSJ wrote manuscript. All authors contributed critically to drafts and gave final approval for publication.

Funding This study was funded by a Royal Society of New Zealand Marsden Fund award (UOA0404) to Kendall Clements and David Raubenheimer. Jethro Johnson was supported by a Commonwealth Scholarship awarded by the Commonwealth Scholarship Commission, UK.

\section{Compliance with ethical standards}

Conflict of interest Jethro Johnson declares he has no conflict of interest. David Raubenheimer declares he has no conflict of interest. Sarah Bury declares she has no conflict of interest. Kendall Clements declares he has no conflict of interest.

Ethical approval Samples were collected under New Zealand Ministry of Fisheries permit 385, and all animal work was carried out following University of Auckland Animal Ethics Committee Approval (\#AEC/03/2006/R456).

\section{References}

Adams NM (1994) Seaweeds of New Zealand an illustrated guide. Canterbury University Press, Christchurch

Anderson MJ, Walsh DCI (2013) PERMANOVA, ANOSIM, and the Mantel test in the face of heterogeneous dispersions: what null hypothesis are you testing? Ecol Monogr 83:557-574. https://doi. org/10.1890/12-2010.1

Baker EJC, Clauss M, Clements KD (2016) Selection and intake of algal species in butterfish (Odax pullus; Labridae). Mar Biol 163:163-168

Behrens MD, Lafferty KD (2007) Temperature and diet effects on omnivorous fish performance: implications for the latitudinal diversity gradient in herbivorous fishes. Can J Fish Aquat Sci 64:867-873. https://doi.org/10.1139/f07-063

Behrens MD, Lafferty KD (2012) Geographic variation in the diet of opaleye (Girella nigricans) with respect to temperature and habitat. PLoS ONE 7:e45901. https://doi.org/10.1371/journ al.pone. 0045901
Bender MG, Pie MR, Rezende EL, Mouillot D, Floeter SR (2013) Biogeographic, historical and environmental influences on the taxonomic and functional structure of Atlantic reef fish assemblages. Glob Ecol Biogeogr 22:1173-1182. https://doi.org/10.1111/ geb.12099

Caut S, Angulo E, Courchamp F (2009) Variation in discrimination factors $\delta^{15} \mathrm{~N}$ and $\delta^{13} \mathrm{C}$ : the effect of diet isotopic values and applications for diet reconstruction. J Appl Ecol 46:443-453

Chiswell SM (1994) Variability in sea surface temperature around New Zealand from AVHRR images. N Z J Mar Freshw Res 28:179-192

Choat JH (1991) The biology of fishes on coral reefs. In: Sale PF (ed) The ecology of fishes on coral reefs. Academic Press, London

Choat JH, Clements KD (1992) Diet in odacid and aplodactylid fishes from Australia and New-Zealand. Aust J Mar Freshw Res 43:1451-1459

Clements KD (1991) Endosymbiotic communities of two herbivorous labroid fishes, Odax cyanomelas and O. pullus. Mar Biol 109:223-229

Clements KD, Bellwood DR (1988) A comparison of the feeding mechanisms of two herbivorous labroid fishes, the temperate Odax pullus and the tropical Scarus rubroviolaceus. Aust J Mar Freshw Res 39:87-107

Clements KD, Choat JH (1993) Influence of season, ontogeny and tide on the diet of the temperate marine herbivorous fish Odax pullus (Odacidae). Mar Biol 117:213-220

Clements KD, Pasch IBY, Moran D, Turner SJ (2007) Clostridia dominate 16S rRNA gene libraries prepared from the hindgut of temperate marine herbivorous fishes. Mar Biol 150:1431-1440. https ://doi.org/10.1007/s00227-006-0443-9

Clements KD, Raubenheimer D, Choat JH (2009) Nutritional ecology of marine herbivorous fishes: ten years on. Funct Ecol 23:79-92

Dalerum F, Angerbjörn A (2005) Resolving temporal variation in vertebrate diets using naturally occurring stable isotopes. Oecologia 144:647-658. https://doi.org/10.1007/s00442-005-0118-0

Ebeling AW, Hixon MA (1991) Tropical and temperate reef fishes: comparison of community structures. In: Sale PF (ed) The ecology of fishes on coral reefs. Academic Press, London

Ferreira CE, Floeter SR, Gasparini JL, Ferreira BP, Joyeux JC (2004) Trophic structure patterns of Brazilian reef fishes: a latitudinal comparison. J Biogeogr 31:1093-1106

Floeter SR, Behrens MD, Ferreira CEL, Paddack MJ, Horn MH (2005) Geographical gradients of marine herbivorous fishes: patterns and processes. Mar Biol 147:1435-1447. https://doi.org/10.1007/ s00227-005-0027-0

Floeter SR, Ferreira EL, Dominici-Arosemena A, Zalmon IR (2004) Latitudinal gradients in Atlantic reef fish communities: trophic structure and spatial use patterns. J Fish Biol 64:1680-1699. https ://doi.org/10.1111/j.1095-8649.2004.00428.x

Folch J, Lees M, Sloane Stanley GH (1957) A simple method for the isolation and purification of total lipides from animal tissues. $\mathrm{J}$ Biol Chem 226:497-501

Franco JN, Wernberg T, Bertocci I, Duarte P, Jacinto D, Vasco-Rodrigues N, Tuya F (2015) Herbivory drives kelp recruits into 'hiding' in a warm ocean climate. Mar Ecol Prog Ser 536:1-9. https://doi. org/10.3354/meps 11445

Gaines SD, Lubchenco JD (1982) A unified approach to marine plantherbivore interactions. II. Biogeography. Annu Rev Ecol Evol Syst 13:111-138

Goldenberg SU, Erzini K (2014) Seagrass feeding choices and digestive strategies of the herbivorous fish Sarpa salpa. J Fish Biol 84:1474-1489. https://doi.org/10.1111/jfb.12371

Gomon MF, Russell BC, Stewart AL, Clements KD (2015) 202. Labridae. Wrasses. In: Roberts CD, Stewart AL, Struthers CD (eds) Fishes of New Zealand. Te Papa Press, Wellington, pp 1365-1405

Gonzalez-Bergonzoni I, Meerhoff M, Davidson TA, Teixeira-de Mello F, Baattrup-Pedersen A, Jeppesen E (2012) Meta-analysis shows 
a consistent and strong latitudinal pattern in fish omnivory across ecosystems. Ecosystems 15:492-503. https://doi.org/10.1007/ s10021-012-9524-4)

Harmelin-Vivien ML (2002) Energetics and fish diversity on coral reefs. In: Sale PF (ed) Coral reef fishes: dynamics and diversity in a complex ecosystem. Elsevier, London, pp 265-274

Hickerson MJ, Cunningham CW (2005) Contrasting quaternary histories in an ecologically divergent sister pair of low-dispersing intertidal fish (Xiphister) revealed by multilocus DNA analysis. Evolution 59:344-360

Hyslop EJ (1980) Stomach contents analysis - a review of methods and their application. J Fish Biol 17:411-429

Iken K, Barrera-Oro ER, Quartino ML, Casaux RJ, Brey T (1997) Grazing by the Antarctic fish Notothenia coriiceps: evidence for selective feeding on macroalgae. Antart Sci 9:386-391. https:// doi.org/10.1017/S0954102097000497

Johnson JS, Clements KD, Raubenheimer D (2017) The nutritional basis of seasonal selective feeding by a marine herbivorous fish. Mar Biol 164:201

Johnson JS, Raubenheimer D, Bury SJ, Clements KD (2012) Effect of ingestion on the stable isotope signatures of marine herbivorous fish diets. J Exp Mar Biol Ecol 438:137-143. https://doi. org/10.1016/j.jembe.2012.09.016

Keith SA, Kerswell AP, Connolly SR (2014) Global diversity of marine macroalgae: environmental conditions explain less variation in the tropics. Glob Ecol Biogeogr 23:517-529. https://doi.org/10.1111/ geb. 12132

Lee RE (2008) Phycology. Cambridge University Press, Cambridge

Longo GO, Ferreira CE, Floeter SR (2014) Herbivory drives largescale spatial variation in reef fish trophic interactions. Ecol Evol 4:4553-4566. https://doi.org/10.1002/ece3.1310

Longo GO, Hay ME, Ferreira CEL, Floeter SR (2018) Trophic interactions across 61 degrees of latitude in the Western Atlantic. Glob Ecol Biogeogr. https://doi.org/10.1111/geb.12806

MacDougall-Shackleton SA (2011) The levels of analysis revisited. Philos Trans R Soc B Biol Sci 366:2076-2085. https://doi. org/10.1098/rstb.2010.0363

Mayr E (1961) Cause and effect in biology. Science 134:1501-1506

McCutchan JH, Lewis WM, Kendall C, McGrath CC (2003) Variation in trophic shift for stable isotope ratios of carbon, nitrogen, and sulfur. Oikos 102:378-390. https://doi.org/10.103 4/j.1600-0706.2003.12098.x

Mora C (2008) A clear human footprint in the coral reefs of the Caribbean. Proc R Soc Ser B 275:767-773. https://doi.org/10.1098/ rspb.2007.1472

Morton J, Miller MC (1973) The New Zealand sea shore. Collins, London

Mountfort DO, Campbell J, Clements KD (2002) Hindgut fermentation in three species of marine herbivorous fish. Appl Environ Microbiol 68:1374-1380. https://doi.org/10.1128/ aem.68.3.1374-1380.2002

Muñoz AA, Ojeda FP (2000) Ontogenetic changes in the diet of the herbivorous Scartichthys viridis in a rocky intertidal zone in central Chile. J Fish Biol 56:986-998. https://doi. org/10.1111/j.1095-8649.2000.tb00887.x

Perga ME, Gerdeaux D (2005) 'Are fish what they eat' all year round? Oecologia 144:598-606. https://doi.org/10.1007/s0044 2-005-0069-5

Poore AG, Campbell AH, Coleman RA, Edgar GJ, Jormalainen V, Reynolds PL, Sotka EE, Stachowicz JJ, Taylor RB, Vanderklift MA, Duffy JE (2012) Global patterns in the impact of marine herbivores on benthic primary producers. Ecol Lett 15:912-922. https://doi.org/10.1111/j.1461-0248.2012.01804.x

Post DM (2002) Using stable isotopes to estimate trophic position: models, methods, and assumptions. Ecology 83:703-718

Reynolds RW, Smith TM, Liu C, Chelton DB, Casey KS, Schlax MG (2007) Daily high-resolution-blended analyses for sea surface temperature. J Clim 20:5473-5496

Ruz CS, Muth AF, Tala F, Pérez-Matus A (2018) The herbivorous fish, Aplodactylus punctatus, as a potential facilitator of dispersal of kelp, Lessonia trabeculata, in Chile. J Exp Mar Biol Ecol 500:112-119. https://doi.org/10.1016/j.jembe.2017.12.007

Shears NT, Babcock RC (2007) Quantitative description of mainland New Zealand's shallow subtidal reef communities. Department for Conservation, Wellington

Stevens CE, Hume ID (1998) Contributions of microbes in vertebrate gastrointestinal tract to production and conservation of nutrients. Physiol Rev 78:393-427

Taylor DI, Schiel DR (2010) Algal populations controlled by fish herbivory across a wave exposure gradient on southern temperate shores. Ecology 91:201-211. https://doi.org/10.1890/08-1512.1

Team RC (2012) R: a language and environment for statistical computing. Team RC, Vienna

Trip ED, Clements KD, Raubenheimer D, Choat JH (2014) Temperature-related variation in growth rate, size, maturation and life span in a marine herbivorous fish over a latitudinal gradient. J Anim Ecol 83:866-875. https://doi.org/10.1111/1365-2656.12183

Trip EDL, Clements KD, Raubenheimer D, Choat JH (2011) Reproductive biology of an odacine labrid, Odax pullus. J Fish Biol 78:741-761. https://doi.org/10.1111/j.1095-8649.2010.02889.x

Vander Zanden MJ, Rasmussen JB (2001) Variation in delta N-15 and delta C-13 trophic fractionation: implications for aquatic food web studies. Limnol Oceanogr 46:2061-2066

Vejrikova I, Vejrik L, Syvaranta J, Kiljunen M, Cech M, Blabolil P, Vasek M, Sajdlova Z, Chung SH, Smejkal M, Frouzova J, Peterka J (2016) Distribution of herbivorous fish is frozen by low temperature. Sci Rep 6:39600. https://doi.org/10.1038/srep39600

Verges A, Doropoulos C, Malcolm HA, Skye M, Garcia-Piza M, Marzinelli EM, Campbell AH, Ballesteros E, Hoey AS, Vila-Concejo A, Bozec YM, Steinberg PD (2016) Long-term empirical evidence of ocean warming leading to tropicalization of fish communities, increased herbivory, and loss of kelp. Proc Natl Acad Sci USA 113:13791-13796. https://doi.org/10.1073/pnas.1610725113

Verges A, Steinberg PD, Hay ME, Poore AG, Campbell AH, Ballesteros E, Heck KL Jr, Booth DJ, Coleman MA, Feary DA, Figueira W, Langlois T, Marzinelli EM, Mizerek T, Mumby PJ, Nakamura Y, Roughan M, van Sebille E, Gupta AS, Smale DA, Tomas F, Wernberg T, Wilson SK (2014) The tropicalization of temperate marine ecosystems: climate-mediated changes in herbivory and community phase shifts. Proc R Soc B Biol Sci 281:20140846. https://doi.org/10.1098/rspb.2014.0846

White TCR (1985) When is a herbivore not a herbivore? Oecologia 67:596-597

Wolf N, Carleton SA, del Rio CM (2009) Ten years of experimental animal isotopic ecology. Funct Ecol 23:17-26. https://doi.org/10 .1111/j.1365-2435.2009.01529.x

Publisher's Note Springer Nature remains neutral with regard to jurisdictional claims in published maps and institutional affiliations. 